This item was submitted to Loughborough's Research Repository by the author.

Items in Figshare are protected by copyright, with all rights reserved, unless otherwise indicated.

\title{
A comparison of stochastic and effective medium approaches to the backscattered signal from a porous layer in a solid matrix
}

PLEASE CITE THE PUBLISHED VERSION

http://dx.doi.org/10.1121/1.3598461

PUBLISHER

(C) Acoustical Society of America

VERSION

VoR (Version of Record)

LICENCE

CC BY-NC-ND 4.0

\section{REPOSITORY RECORD}

Pinfield, Valerie J., Richard E. Challis, and Robert A. Smith. 2012. "A Comparison of Stochastic and Effective Medium Approaches to the Backscattered Signal from a Porous Layer in a Solid Matrix". figshare. https://hdl.handle.net/2134/11084. 
This item was submitted to Loughborough's Institutional Repository (https://dspace.lboro.ac.uk/) by the author and is made available under the following Creative Commons Licence conditions.

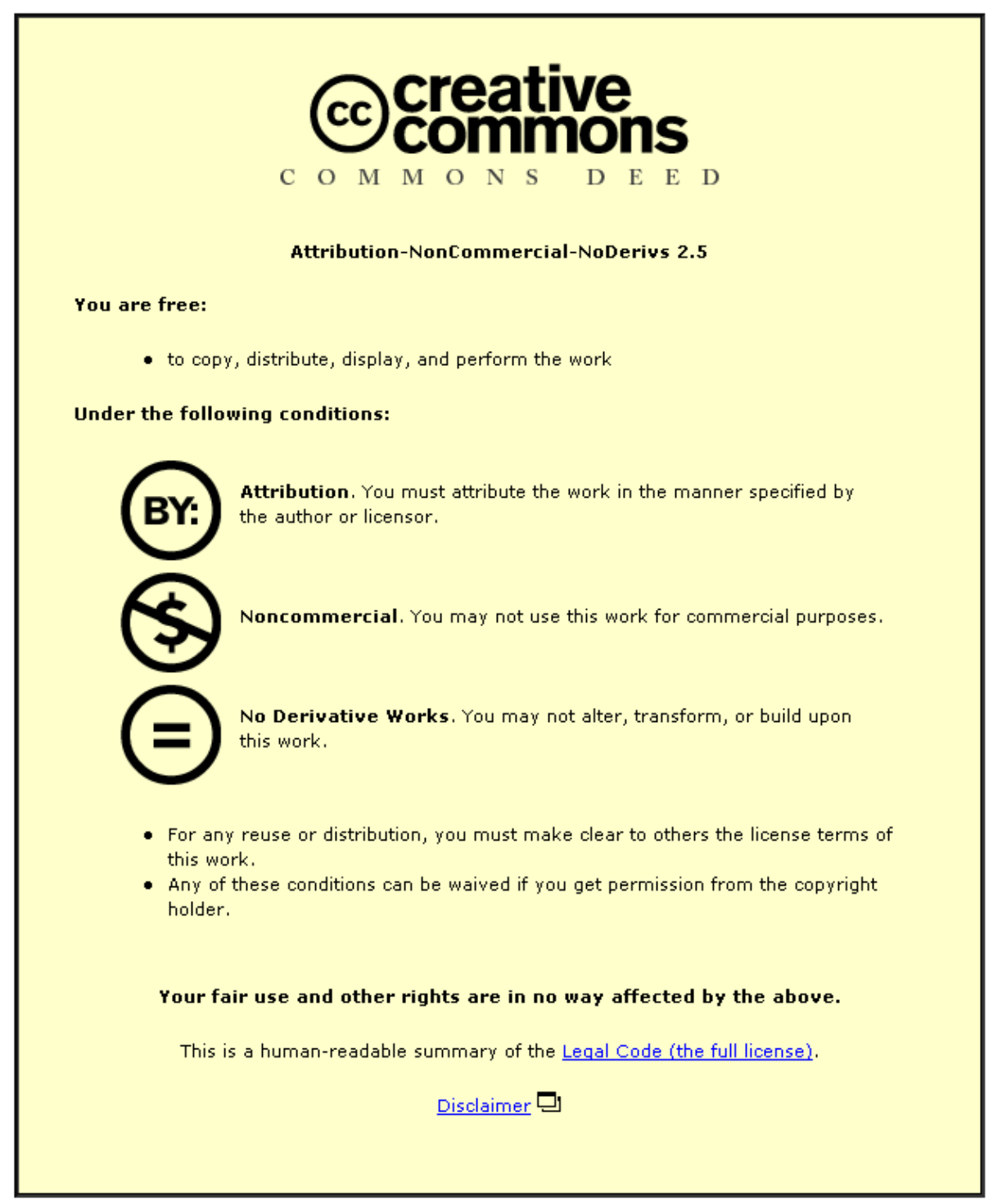

For the full text of this licence, please go to: http://creativecommons.org/licenses/by-nc-nd/2.5/ 


\title{
A comparison of stochastic and effective medium approaches to the backscattered signal from a porous layer in a solid matrix
}

\author{
Valerie J. Pinfield ${ }^{\text {a) }}$ and Richard E. Challis \\ Electrical Systems and Optics Division, Faculty of Engineering, University of Nottingham, \\ University Park, Nottingham, NG7 2RD United Kingdom \\ Robert A. Smith \\ QinetiQ Ltd., Cody Technology Park, Ively Road, Farnborough, Hampshire GU14 OLX, United Kingdom
}

(Received 7 April 2010; revised 15 April 2011; accepted 17 May 2011)

\begin{abstract}
This paper reports a study of the backscattering behavior of a solid layer containing randomly spaced spherical cavities in the long wavelength limit. The motivation for the work arises from a need to model the responses of porous composite materials in ultrasonic NDE procedures. A comparison is made between models based on a summation over discrete scatterers, which show interesting emergent properties, and an integral formulation based on an ensemble average, and with a simple slab effective medium approximation. The similarities and differences between these three models are demonstrated. A simple quantitative criterion is established which sets the maximum frequency at which ensemble average or equivalent homogeneous medium models can represent echo signal generation in a porous layer for given interpore spacing, or equivalently, given pore size and concentration. (C) 2011 Acoustical Society of America. [DOI: 10.1121/1.3598461]
\end{abstract}

PACS number(s): $43.35 \mathrm{Cg}, 43.35 \mathrm{Zc}$ [PEB]

Pages: $122-134$

\section{INTRODUCTION}

The rapidly increasing use of fiber reinforced composites, particularly in the aerospace sector, brings with it requirements for nondestructive evaluation (NDE), which is likely to be based on ultrasonic pulse-echo scanning. Development, formal evaluation, and understanding of these techniques requires mathematical/computational models of ultrasonic wave propagation in stratified structures in which each layer is a multiphase medium. Many such models exist in the literature, ${ }^{1-3}$ and these have their basis in earlier work in geophysical imaging. ${ }^{4,5}$ The mechanical properties of the individual layers in such models are, typically, based on equivalent medium theories for mixtures of fiber, resin, and sometimes porous inclusions. ${ }^{6-8}$ Here, porosity is significant because it reduces the shear strength of the composite approximately in proportion to its volume concentration, at least at low concentrations. It is therefore important that porosity is detectable and quantifiable in NDE procedures. An early demonstration of such detection was achieved by Nair et al..$^{9}$ and Adler et al. ${ }^{10}$ who used the additional attenuation due to scattering from cavities to determine porosity in a solid material. More recently a basic porosity detection scheme was developed for layered composites which incorporated porosity-induced attenuation into the layered propagation model on the basis of scattering theory. ${ }^{11}$

Historically, porosity was incorporated into equivalent medium models for fiber-reinforced composites for engineering applications by use of the elastostatic case, in which its effect is to reduce the density and elastic moduli of the resin component; ${ }^{6-8}$ as such, results were only valid

\footnotetext{
a) Author to whom correspondence should be addressed. Electronic mail: valerie.pinfield@nottingham.ac.uk
}

in the very long wavelength limit. Somewhat separately from these developments which were tailored to engineering applications, methods were introduced by key workers such as Eshelby, ${ }^{12}$ Hill $^{13}$ and Budiansky, ${ }^{14}$ to obtain effective elastic properties for composite materials in the static case. The limits within which the effective elastic moduli must exist were defined over a wide concentration range by the so-called Hashin-Shtrikman bounds. ${ }^{15}$ Later, the search for effective properties was extended to the elastodynamic case by a number of schemes, termed homogenization methods by Parnell et al. ${ }^{16}$ These schemes include the selfconsistent and effective medium methods for which a substantial literature may be found, among them the work of Sabina and Willis ${ }^{17}$ and Kanaun and Levin; ${ }^{18}$ further examples are cited by Kim. ${ }^{19}$ Although variants on the models exist, these methods often consider each inclusion or scatterer to be located in a homogeneous medium which has the effective properties of the material; the average scattered field from all such inclusions must vanish, by selfconsistency arguments. Application of this criterion leads to determination of the effective properties of the material. An alternative homogenization scheme has been proposed by Parnell and co-workers, ${ }^{16}$ which adopts an integral equation approach, using the static Eshelby tensor ${ }^{12}$ to relate the strain in the inclusion to that in the matrix. A useful comparison of the wave propagation parameters derived from various effective medium models and multiple scattering theories has been carried out by Kim, ${ }^{19}$ for the two-dimensional case of randomly distributed circular cylinders. In general, the homogenization literature has been concerned with the derivation of effective elastic properties, and the resulting wave propagation parameters, but not with the reflected and transmitted waves which are of interest in the present work. 
A second body of literature on the determination of effective properties of inhomogeneous materials adopts scattering theory, based on the foundational studies by Foldy, ${ }^{20}$ $\mathrm{Lax}^{21,22}$ Twersky, ${ }^{23}$ Waterman and co-workers, ${ }^{24,25}$ and Varadan. ${ }^{26}$ In these studies, the scattered fields from each scatterer are summed, and then ensemble-averaged over all possible (usually) random configurations of scatterer positions. A solution to the equations can only be obtained by making an assumption about the incident field at each scatterer-the so-called closure assumption. The most common approximations are those due to Foldy ${ }^{20}$ (the Foldy closure approximation) and later $\operatorname{Lax}^{21,22}$ (the quasicrystalline approximation, QCA), still widely adopted in both the acoustic and electromagnetic scattering literature. While the effective wavenumber due to scattering theory is well-established for dilute systems, its application to more concentrated systems is still the subject of development. However, there has been a recent emergence of interest in the effective properties of inhomogeneous materials derived from scattering theory; in particular a number of workers ${ }^{27-33}$ have attempted to obtain properties other than the elastic moduli, such as effective density, effective viscosity and the effective reflection and transmission coefficients of both a semiinfinite half-space, and a slab.

A number of recent papers have presented new formulations of multiple scattering theory, based on the earlier models, in order to obtain effective wavenumber and other properties, in particular systems. They follow either the Waterman and Truell ${ }^{24,27}$ or Fikioris and Waterman ${ }^{25,28,29}$ formulations, expressing the scattered field either as a multipole expansion, based on the Rayleigh partial-wave method, ${ }^{27,28}$ or through the transition-matrix description of Varadan. ${ }^{26,29}$ While two of the studies apply to nonviscous fluids, ${ }^{27,29}$ the other is applicable to cylinders in solids, ${ }^{28}$ considering both longitudinal and shear wave propagation. Maurel $^{30}$ took a slightly different approach, applying the Born approximation to terminate the scattering field equations, rather than applying a closure assumption, and using a Green's function description rather than the multipole expansion for the scattered fields. Linton and Martin $^{34}$ have shown a new derivation of the effective wavenumber, using a multipole expansion and following Foldy, ${ }^{20}$ with the Lax QCA $;^{21,22}$ they confirm the results obtained by Fikioris and Waterman, ${ }^{25}$ and Lloyd and Berry. ${ }^{35}$ Based on these newer effective wavenumber derivations, or on the older versions, various workers have identified a set of effective properties for the material, for example, density, modulus, and viscosity. ${ }^{31-33}$

Of greater interest in the present work, is the determination of the reflection characteristics of a layer, or slab, of material containing scatterers, which has been derived by a number of the same workers, based on the scattering theory formulations described above. ${ }^{27-30,36}$ In summary, what has been established is as follows: (a) The effective material properties of a slab or layer are the same as the effective material properties of a half-space. (b) The effective (ensembleaveraged) reflected and transmitted fields from the slab have the same form as the summed multiple reflections from a homogeneous layer. ${ }^{27,30}$ (c) The effective reflection and trans- mission coefficients define an effective impedance which relates to the effective density and wavenumber of the material in the same way as for a homogeneous material. ${ }^{29,31,32}$ While some of these works are applicable to cylinders, and some only to nonviscous fluids, these principles appear to be of general applicability.

The scattering theory formulations described in the previous paragraphs define effective properties for a material containing scatterers, taking a statistical average over scatterer positions (ensemble average). The result, an effective impedance relating to the scatterer (cavity) properties, is convenient for implementation in models of layered composites. However, in an experimental measurement, a snapshot is taken with a single realization of scatterer locations. Although some reduction in the incoherent field may be achieved by use of a large area transducer, the snapshot still pertains only to one sample of scatterer locations (for a solid matrix). Hence, in this paper we wish to investigate the conditions in which an ensemble-average model is a valid description of the reflected signal obtained in a single snapshot experimental measurement, and to establish the validity of the ensemble-average models by numerical modeling. By stochastic modeling, and by taking a simple ensemble-average using numerical integration, we explore the emergence of the effective properties of a matrix containing cavities, and confirm the validity of the effective properties obtained from published ensemble averaged scattering models. Few numerical studies of the scattered field response for a layer of scatterers exist; the one-dimensional computation of Maure ${ }^{30}$ and the finite-difference time domain simulation of Dubois et $a l .{ }^{37}$ validate their ensemble-average formulation, but no investigation was made of the application to a single snap-shot measurement.

Our models are each based on the multipole expansion, partial-wave method for the scattering by a single spherical cavity, originating in the work of Rayleigh. ${ }^{38}$ The method is based on an analysis of incident and scattered waves in terms of partial wave modes; the scattered amplitude of each mode is obtained by the application of boundary conditions at the surface of the obstacle. The basic method was developed and updated to the current commonly used form by Epstein and Carhart, ${ }^{39}$ Allegra and Hawley, ${ }^{40}$ and Ying and Truell. ${ }^{41}$ The three formulations pertained to different physical states of the scattering object and surrounding media, namely, fluid in fluid, solid in fluid and any material in solid (neglecting thermal effects) respectively. The relationships between them have been discussed by Challis et al., ${ }^{42}$ see also Challis et $a .^{43}$ Our numerical models use these scattered fields, either for individual scatterers summed or averaged as appropriate, or to obtain the ensemble-averaged effective properties of the layer.

In summary, we aim to establish the validity of the ensemble-average results for the effective properties of a layer containing cavities by numerical experiment, and to investigate the emergence of the effective properties from the incoherent wave fields. In order to address these issues we imagine the arrangement shown on Fig. 1 (top half), which illustrates a layer of spherical cavities embedded in a solid (e.g., resin) and which scatter signals back to an 


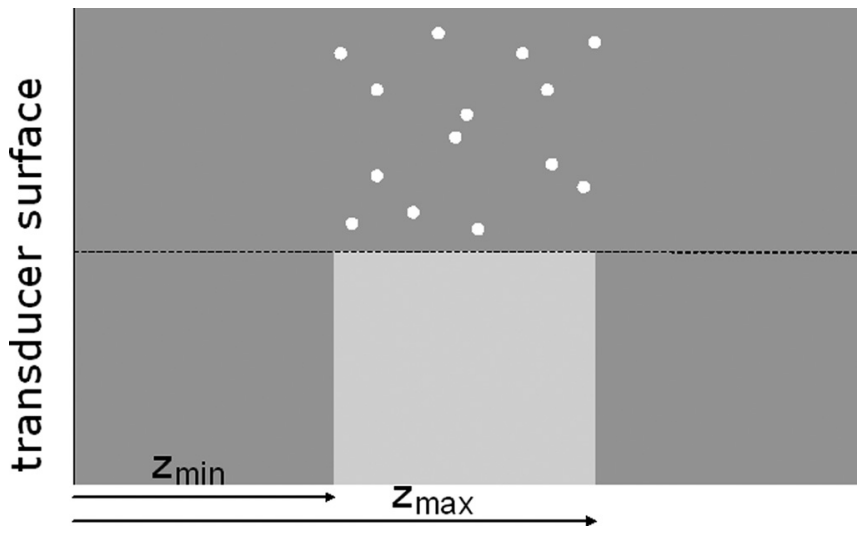

FIG. 1. System configurations for the discrete scatterer model A and ensemble average model B (both in top half, above dashed line), and effective medium model C (bottom half, below dashed line). For models A and B (top half, above dashed line), spherical cavities are embedded in solid material in the region $z_{\min }<z<z_{\max }$. For the effective medium model (bottom half, below dashed line) a homogeneous solid material is present in the region $z_{\min }<z<z_{\max }$. In both cases, the transducer is directly in contact with the medium in the region $z<z_{\min }$ which has the same properties as the matrix surrounding the cavities, and the material in the region $z>z_{\max }$.

interrogating transducer. We compare representations of this arrangement by three related models. The first (Model A, Sec. II A) is a stochastic one which simulates a single realization of cavity positions, such as would be found in an experimental sample. The second (Model B, Sec. II B) provides a simple ensemble averaged response, obtained numerically by integrating over the scatterer positions of Model A. We simulate a range of concentrations of scatterers in order to explore the conditions under which the ensemble averaged result represents the single configuration of model A. The third (Model C, Sec. II C) represents the layer of scattering cavities by an equivalent homogeneous medium (Fig. 1, bottom half) with properties derived by previous workers. Our numerical experiment provides a validation of the effective medium formulation by comparison of the numerically simulated ensemble-averaged results with the effective medium model. However, our primary aim here is to investigate the conditions under which such a formulation can represent an ultrasonic echo signal that might be obtained in a single snap-shot measurement. The results of the models are investigated and compared in Sec. V, and the conditions for validity of the effective medium description are discussed in Sec. VI.

\section{THE MODELS}

\section{A. Discrete scatterer model A}

We consider the scattered field received from a number of cavities located in a region of the material, those cavities having fixed positions. We investigate how that received signal is affected by the number or concentration of cavities present, and under what conditions the signal approaches that reflected from a homogeneous material. The configuration under consideration for the scattering model was shown in the top half of Fig. 1. A transducer is coupled directly to a solid medium, in which a number of spherical cavities are distributed in the region $z_{\min }$ to $z_{\max }$ from the transducer (parallel to the axis of the transducer). There is assumed to be no "interface" either between the transducer and the medium, or between the homogeneous region and the region of scatterers. In the latter case, the absence of an interface is due to the fact that the matrix surrounding the scatterers is assumed to have the same properties as the homogeneous region. Its wavenumber $k=\omega / c(\omega)+i \alpha(\omega)$, where $c(\omega)$, $\alpha(\omega)$ are the wave speed and the attenuation respectively and $\omega$ is the angular frequency. The transducer is assumed to be of infinite extent to avoid complications due to diffraction at the transducer, and its response is considered to be proportional to the normal displacement at a point on the surface of the transducer. The incident field on the system is a plane wave of infinite lateral extent. We obtain the transducer response to the signals scattered by the cavities by summing the normal displacement at a point on the transducer resulting from the scattered field from the individual cavities.

\section{Scattering coefficients for a spherical cavity}

We adopt the Rayleigh method to obtain the scattered wave potential for the propagational mode resulting from a planar incident wave in the $z$ direction; it is given by a sum over all scattered partial wave modes, ${ }^{38-42}$ thus

$$
\phi_{R}=\sum_{n=0}^{\infty} i^{n}(2 n+1) A_{n} h_{n}(k r) P_{n}(\cos \theta)
$$

using spherical polar coordinates $(r, \theta, \varphi)$ with origin at the center of the scatterer. Note that the convention $i=\sqrt{-1}$ has been used throughout. $h_{n}$ is a spherical Hankel function of the first kind determining the radial distributions, and $P_{n}$ are the Legendre polynomials, representing the angular distributions, $A_{n}$ is the scattering coefficient for the $n$th partial wave order. The result is written in spherical polar coordinates with origin at the center of the scatterer. In the long wavelength region, for which the wavelength of the propagational mode is considerably longer than the radius of the obstacle, only the first few orders are significant, typically up to $n=2$.

For the specific case of interest in the current work, i.e., cavities in a solid matrix, simplification of the model is possible: no wave modes exist inside the cavity and the stress at the surface must be zero. As a further simplification, the thermal effects can be neglected since these are generally small in solid systems. The coefficients can be derived from the generalized matrix equation set out in Ref. 42 and described in Ref. 43. [It should be noted that the matrix Eq. (9) of Ref. 43 has a missing minus sign multiplying the right hand side of the matrix equation.] However, at low frequency (where both longitudinal and shear wavelengths are long compared with the cavity radius), simplified solutions can be obtained for the scattering coefficients. Ying and Truell's ${ }^{41}$ results for the zero, first and second order coefficients have been derived in updated notation using the Maple algebraic software ${ }^{44}$ [and correcting a factor of -1 in the original paper, Eq. (34) of Ref. 41], thus 


$$
\begin{aligned}
& A_{0}=i(k a)^{3} \frac{\left(1-4 \hat{c}^{2} / 3\right)}{4 \hat{c}^{2}} \approx \frac{2}{3} i(k a)^{3}, \\
& A_{1}=-\frac{1}{9} i(k a)^{3}, \\
& A_{2}=i(k a)^{3} \frac{4 \hat{c}^{2}}{3\left(9-4 \hat{c}^{2}\right)} \approx \frac{1}{24} i(k a)^{3},
\end{aligned}
$$

where $\hat{c}=k / k_{S}$ is the ratio of shear wave speed to compressional wave speed. The approximated results have been obtained by using the assumption $\hat{c} \approx 1 / 2$. The coefficients are dominated by the imaginary part (relating to sound speed); the smaller real part (which affects attenuation) results from the imaginary part (intrinsic attenuation) of the wavenumber $k$. In physical terms, the scattering coefficients can be understood to relate to the monopole, "breathing" motion of the cavity $\left(A_{0}\right)$, and the variation of the local field around the surface of the cavity which affects its motion at dipole and higher orders.

\section{Incident field}

The wave transmitted by the transducer is assumed to be a longitudinal plane wave of infinite lateral extent propagating in the positive $z$ direction, in common with many studies of effective medium properties. Using a scalar displacement potential $\phi$ for longitudinal waves of the form $\mathbf{u}=-\nabla \phi$ (where $\mathbf{u}$ is the displacement), the incident field at any location is given by

$$
\phi_{\text {inc }}(\omega)=\Phi_{i} e^{i k z}
$$

We have adopted the $e^{-i \omega t}$ convention for time-dependence, in accordance with Refs. 39, 40, 42, and the majority of physical acoustics references. The corresponding timedomain description is obtained by Fourier transformation. In this convention, the Fourier transform is defined by $F(\omega)=\int f(t) e^{i \omega t} d t$, and the frequency-domain representation of a time delay is $e^{+i \omega t}$. It should be noted that this is the opposite sense from the engineering convention for Fourier transforms, including those implemented in MATLAB. The amplitude $\Phi_{i}$ has dimensions of length squared.

The exciting wave at any scatterer is assumed to be identical to the incident wave, Eq. (5); no modification of the incident wave is made to incorporate the scattered fields from other scatterers. This is a lower order approximation than that of Foldy, ${ }^{20}$ who assumed that the exciting field was equal to the total ensemble-averaged field in the medium and Lax, ${ }^{21,22}$ who assumed that the exciting field with one scatterer fixed was the same as that with two scatterers fixed (the quasicrystalline approximation). We make our assumption for simplification of the numerical simulation; inclusion of scattered fields by all other scatterers would add considerable complexity to the simulation. The assumption does, however, constrain our results to low concentrations, although we simulate up to $20 \%$ concentration under this assumption to explore the emergence of the effective medium properties.
When the transducer is transmitting or receiving, we assume that its response is proportional to the normal displacement at the transducer face (at $z=0$ ), which, for the incident field, is

$$
\mathbf{u}_{z, \text { inc }}=-\left.\frac{\partial \phi}{\partial z}\right|_{z=0}=-i k \Phi_{i} .
$$

In the numerical calculations which follow, we have simulated a typical transducer response by multiplication (in the frequency domain) by an experimentally-measured pulseecho response obtained in water.

\section{Field at the transducer: Received signal}

We assume that the field incident on the scatterer is planar, propagating in the $z$ direction, and identical to the transmitted wave for all scatterers. Moreover, we now assume that the transducer is a "large" distance from the scatterer (greater than a wavelength), so that the scattered field can be expressed in its far-field form

$$
\phi \rightarrow f(\theta) \frac{e^{i k r}}{r} \text { as } r \rightarrow \infty
$$

for unit incident field. ${ }^{45}$ The scattering amplitude $f(\theta)$ defines the angular variation of the scattered field, and is independent of the azimuthal angle in this case. It is related to the scattering coefficients of the partial wave orders, $A_{n}$ (defined in Sec. II A 1), Eq. (1) as follows:

$$
f(\theta)=\frac{1}{i k} \sum_{n=0}^{\infty}(2 n+1) A_{n} P_{n}(\cos \theta) .
$$

We now consider the normal displacement (which determines the transducer response) of the scattered field received at a point $O$ on the transducer surface from a single scatterer located at cylindrical coordinates $(R, z)$ relative to $O$. First, we evaluate the field at a point $Q$ located at $(0, \zeta)$ (see Fig. 2), second obtain the normal displacement by taking the derivative of the potential with respect to the receiving point position, $\zeta$, with the scatterer position fixed, and then evaluate at $\zeta=0$ to obtain the normal displacement at the transducer surface.

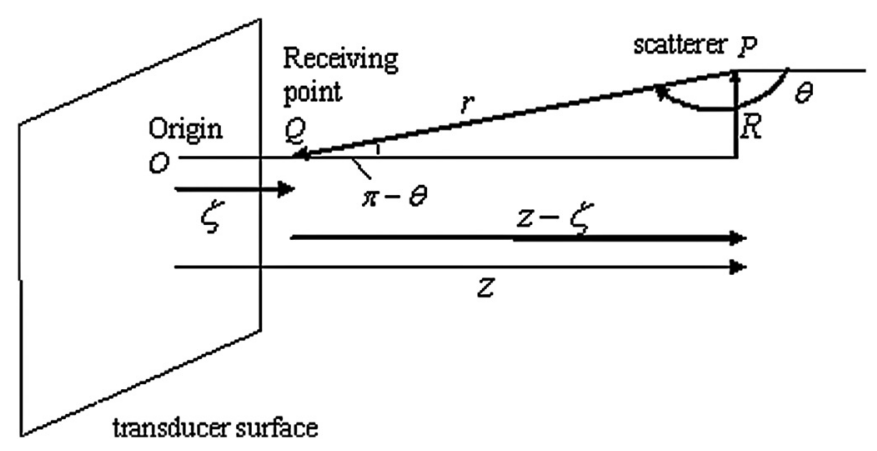

FIG. 2. Configuration for the derivation of the normal displacement at the transducer from a single scatterer in the discrete scatterer model A and ensemble average model B. 
The potential of the scattered wave at the point $Q$ is given by

$$
\phi_{Q, s g l e}=\Phi_{i} e^{i k z} f(\theta) \frac{e^{i k r}}{r}
$$

with

$$
\begin{aligned}
& r^{2}=R^{2}+(z-\zeta)^{2}, \\
& \theta=\pi-\cos ^{-1}\left(\frac{z-\zeta}{r}\right),
\end{aligned}
$$

so that the normal displacement at the transducer due to a single scatterer at $(R, z)$ is given by

$$
\begin{aligned}
\mathbf{u}_{z, \text { sgle }}= & \left(-i k \Phi_{i}\right) e^{i k z} \cdot \frac{e^{i k r_{0}}}{i k r_{0}^{2}} \\
& \times\left[-\left.\frac{\partial f(\theta)}{\partial \theta}\right|_{\theta_{0}} \sin \theta_{0}-i k z \cdot f\left(\theta_{0}\right)\left\{1-\frac{1}{i k r_{0}}\right\}\right],
\end{aligned}
$$

where the subscript 0 denotes evaluation at the position $\zeta=0$. The reflection coefficient, defined as the ratio of the normal displacement of the received and transmitted signals, at the front of the scatterer region,

$$
R_{\text {ref }}=\frac{\mathbf{u}_{z, \text { rec }}}{\mathbf{u}_{z, \text { inc }}} e^{-2 i k z}
$$

is given by

$$
\begin{aligned}
R_{\text {sgle }} & =e^{-2 i k z} \frac{\mathbf{u}_{z, \text { sgle }}}{\mathbf{u}_{z, \text { inc }}} \\
& =\frac{e^{i k\left(r_{0}-z\right)}}{i k r_{0}^{2}}\left[-\left.\frac{\partial f(\theta)}{\partial \theta}\right|_{\theta_{0}} \sin \theta_{0}-i k z \cdot f\left(\theta_{0}\right)\left\{1-\frac{1}{i k r_{0}}\right\}\right] .
\end{aligned}
$$

The response has a number of features which are worth observing. The propagation delay combines the outward distance and inward distance, to give a phase shift which depends on both $z$ and $R$ coordinates of the scatterer. All terms scale with scatterer-transducer distance faster than $1 / r$ which implies that the signal received from the scatterer decreases as the scatterer moves to a larger radial coordinate position. This is partly due to the fact that the signal arrives at the transducer at an increasingly oblique angle as $R$ increases, so that the component of displacement normal to the transducer is reduced. For isotropic scatterers, the first term in the brackets vanishes.

Where there are many scatterers located in the region, the scattered fields are summed at the transducer giving

$$
\begin{aligned}
\mathbf{u}_{z, \text { mult }}= & \left(-i k \Phi_{i}\right) \sum_{j=1}^{N_{s c}} e^{i k z_{j}} \cdot \frac{e^{i k r_{j}}}{r_{j}^{2}} \\
& \times\left[-\left.\frac{\partial f(\theta)}{\partial \theta}\right|_{\theta_{j}} \sin \theta_{j}-i k z_{j} \cdot f\left(\theta_{j}\right)\left\{1-\frac{1}{i k r_{j}}\right\}\right],
\end{aligned}
$$

where the subscript $j$ denotes an individual scatterer. We term this the discrete scatterer solution, model A.

Although effective medium properties are frequently derived for slabs of infinite lateral extent, for the purposes of numerical simulation the problem must be constrained to a finite region. Hence, we consider a domain defined by the coordinates $z_{\min } \leq z \leq z_{\max }, R \leq R_{\max }$ in which centers of the scatterers are located. The decay of the signal strength with radial coordinate $R$ for single scatterers implies that a slab of infinite lateral extent may be approximated by such a finite domain - provided that the radius of the domain containing scatterers is chosen to be sufficiently large that the contribution from scatterers located at larger $R$ is negligible.

\section{B. Ensemble average model B}

Effective medium properties are often derived by taking an ensemble average over scatterer positions, whereas the result given above represents the signal received from a single realization of scatterer positions. Since we are interested in both the single-realization case, and the emergence of effective medium behavior, we now derive the corresponding ensemble-average result for identical scatterers which are randomly distributed in the region. For simplicity, the scatterers are treated as points for geometrical purposes, allowing use of a uncorrelated, uniform distribution for their locations, but retain the scattering properties of a sphere of given radius. Such approximations are common in the effective medium literature. ${ }^{27,30}$ Taking the sum over all scatterers from Eq. (13), using volume elements $d V$ $=2 \pi R d R d z=2 \pi r d r d z$ the ensemble average is given by

$$
\mathbf{u}_{z, \text { ave }}=\int_{z=z_{\min }}^{z_{\max }} \int_{r=z}^{\infty} 2 \pi N \mathbf{u}_{z, \text { sgle }} r d r d z
$$

with a number density $N$, and dropping the subscripts from Eq. (10). This ensemble average is not rigorously derived but is obtained as a simple single-scattering, low concentration extension to the multiple-discrete scatterer result, by integrating over random, uncorrelated scatterer positions. It is made possible by the assumption that the exciting field at each scatterer is identical to the incident field from the transducer. Under that approximation, the order in which the spatial derivative (for normal displacement) and the integration (for the ensemble average) are taken does not affect the result; this is not necessarily the case in a full statistical treatment under the Foldy or Lax closure approximations. Numerical simulation will be used to establish its validity as the limit of the multiple discrete scatterer formulation and its correspondence with the effective medium model. For numerical simulation, the limits of the integral over $r$ must be finite, and as for the discrete scatterer model the region is defined by the limit $R \leq R_{\max }$. The decrease in the magnitude of the normal displacement for scatterers located as $R$ increases is faster than $1 / r$, resulting in a smaller contribution from ring elements of larger radius even accounting for the greater number of scatterers in those areas $(2 \pi r N d r d z)$. Hence it is possible to approximate the integral by a finite region $R \leq R_{\max }$. 


\section{Effective medium model C}

Thus far we have established the solution for the signal backscattered by a single cavity (scatterer) and by multiple discrete cavities (model A). We have also taken the ensemble averaged response of the region under the same formulation (model B). Our final model (model C) treats the region containing scatterers as an equivalent homogeneous medium (see Fig. 1, bottom half), with properties defined by an effective medium model published in the literature. ${ }^{27,31,32}$ We term this the effective medium modelmodel C. Our aim is to confirm the validity of this effective medium model by comparison within numerical simulations with the discrete scatterer and ensemble average models A and B. Our simulations will also explore the emergence of the effective medium behavior to establish the conditions under which it may be applied in typical measurement situations.

The configuration considered in the effective medium model is shown in the bottom half of Fig. 1. Whereas for the discrete scatterer and ensemble-averaged models A and B (Fig. 1, top half), the scatterers were embedded in a matrix identical to the scatterer-empty region and there were no interfaces in the system, now we consider the equivalent homogeneous medium in the region previously occupied by the scatterers, and with interfaces at the front and back of the region. No individual scatterers are considered to be present in the effective medium.

For a homogeneous medium, the signal received at the transducer can be simply written in terms of the reflection and transmission at each interface, accounting for multiple reflections in the layer, ${ }^{46}$

$$
\mathbf{u}_{z, \mathrm{eff}}(\omega)=e^{2 i k z_{\min }} \cdot r_{12}\left[1-t_{12} t_{21} e^{2 i k d} H(\omega)\right],
$$

where $r_{i j}, t_{i j}$ are the displacement reflection and transmission coefficients at the interface from medium $i$ to medium $j$, $d=z_{\max }-z_{\min }$ is the thickness of the layer and the multiple reverberation term is

$$
H(\omega)=\left[1-r_{12}^{2} e^{2 i k d}\right]^{-1} .
$$

If the ratio of the impedance in the layer to the solid matrix is $\hat{Z}$ then

$$
r_{12}=-\frac{\hat{Z}-1}{\hat{Z}+1}, \quad t_{12}=\frac{2 \hat{Z}}{\hat{Z}+1}, \text { and } \quad t_{21}=\frac{2}{\hat{Z}+1} .
$$

Note that the reflection coefficient is defined with the opposite sign to that in Ref. 46; it is specified here in accordance with the definition of reflection coefficient given in Eq. (11). The impedance of a medium is usually expressed in the form

$$
Z=\rho c
$$

with density $\rho$, and longitudinal sound speed, $c$.

A number of workers have shown that the effective, ensemble-averaged reflected field from a layer of scatterers, takes the same form as that given above for a homogeneous layer, ${ }^{27,29,30,36}$ with a generalized definition for the effective impedance of

$$
Z=\frac{\omega \rho_{\text {eff }}}{K_{\text {eff }}}
$$

although Le Bas et al. ${ }^{29}$ retain the original definition, using the real part of the effective wavenumber in the denominator (to obtain effective wave speed). Since we are working at low concentrations, we work to first order in volume fraction only, taking the Foldy ${ }^{20}$ result for the effective, ensembleaveraged wavenumber, $K$,

$$
\left(\frac{K^{2}}{k^{2}}\right)=1+\frac{3 \phi}{k^{2} a^{3}} f(0)
$$

where $\phi$ is the volume fraction of cavities and $k$ is the wavenumber in the matrix. The effective density, derived from ensemble-averaged scattering theory models, ${ }^{27,31,32}$ can be written to first order as

$$
\rho_{\mathrm{eff}}=\rho\left[1+\frac{3 \phi}{2 k^{2} a^{3}}\{f(0)-f(\pi)\}\right]
$$

which, using the results for cavities from Sec. II A [Eqs. (8) and (3)] simplifies in the long wavelength limit to

$$
\rho_{\text {eff }}=\rho\left[1+\frac{3 \phi}{2 k^{2} a^{3}}\left\{\frac{6 A_{1}}{i k}\right\}\right] \approx \rho(1-\phi) .
$$

This is equivalent to the static case, and also agrees with the effective density derived by Parnell et al. by an alternative homogenization scheme. ${ }^{16}$ Thus, for our effective medium model, we adopt an effective impedance, Eq. (19), using the Foldy effective wavenumber, Eq. (20) and the effective density given by the right hand side of Eq. (22).

The impedance ratio of the effective medium corresponding to randomly distributed cavities is therefore

$$
\begin{aligned}
\hat{Z} & =\frac{Z_{\mathrm{eff}}}{Z} \approx(1-\phi) \frac{k}{K} \approx(1-\phi)\left(1-\frac{39}{48} \phi\right) \\
& \approx 1-\frac{87}{48} \phi
\end{aligned}
$$

at low frequency, and low concentration of cavities. Clearly the impedance ratio is independent of frequency at the lowest frequencies.

\section{ANALYTICAL COMPARISON OF MODELS}

Before we discuss the numerical simulations using the three models, we establish the analytical correspondence between our ensemble average of the discrete scatterer model (model B) and the published effective medium models, adopted in our model C. Many effective medium studies focus on isotropic scatterers, since this simplifies the formulation greatly, and we take the same restriction here to obtain an analytical solution to Eq. (14) for our ensemble averaged result. However, isotropic scattering implies that only the 
zero-order scattering coefficient is nonzero, which is only possible for scatterers whose density is equal to that of the surrounding medium. Hence, for the purposes of this comparison, the scatterers are no longer considered to be cavities, which cannot be isotropic scatterers, but to be scattering objects whose density is matched to that of the surrounding medium.

First, we consider the reflection coefficient for the effective medium for a semi-infinite half-space, using isotropic, density-matched scatterers, in Eqs. (17) and (23), obtaining

$$
R_{\mathrm{eff}, \text { iso }, \infty} \approx \frac{K-k}{K+k} \approx \frac{3 \phi f_{\text {iso }}}{4 a^{3} k^{2}}=\frac{\pi N}{k^{2}} f_{\text {iso }}
$$

in accordance with Parnell et al. ${ }^{16}$ [Eq. (4.10)]. For the layer, the effective medium model with isotropic scatterers has a reflection coefficient of

$$
R_{\text {eff }, \text { iso }} \approx \frac{\pi N}{k^{2}} f_{\text {iso }}\left[1-e^{2 i K d}\right]
$$

using Eqs. (23), (17), (15). These results are all obtained using the effective impedance from the published ensembleaverage scattering models (see previous section for details).

Now we derive the equivalent results for our ensembleaveraged discrete scatterer model, Eq. (14) for densitymatched, isotropic scatterers.

$$
\begin{aligned}
\mathrm{R}_{\mathrm{ave}, \text { iso }}= & e^{-2 i k z_{\min }} \frac{\mathbf{u}_{z, \text { ave }}}{\mathbf{u}_{z, \text { inc }}} \\
= & 2 \pi N e^{-2 i k z_{\min }} \int_{z=z_{\min }}^{z_{\max }} \int_{r=z}^{\infty}(-i k z) f_{\text {iso }} \\
& \times e^{i k z} \frac{e^{i k r}}{r}\left[1-\frac{1}{i k r}\right] d r d z \\
\mathrm{R}_{\text {ave }, \text { iso }}= & 2 \pi N f_{\text {iso }} e^{-2 i k z_{\min }} \int_{z=z_{\min }}^{z_{\max }}\left[\frac{e^{2 i k z}}{i k}\right] d z \\
= & \frac{\pi N f_{\text {iso }}}{k^{2}}\left[1-e^{2 i k d}\right]
\end{aligned}
$$

and for an infinite half-space $\left(z_{\max } \rightarrow \infty\right)$ only the first term in the square bracket remains, the second being the echoes from the distal boundary of the layer. A comparison of Eqs. (26) and (25) shows that the reflection coefficient obtained from our far-field scattering ensemble-averaged model is almost identical to that obtained from published effective medium models for isotropic scatterers. ${ }^{27,31,32}$ The difference is in the propagation speed across the layer, which in this case is expressed through the wavenumber of the matrix medium, $k$ and in the effective medium models is given through the effective wavenumber of the scattering medium, $K$. The latter is the more realistic result, and the difference probably arises in the approximation of uniform incident field, and that the scattered waves travel only through the matrix medium in our model. However, the correspondence of the two models builds confidence in the discrete scatterer formulation as a means of validating the effective medium models by numerical simulation.

\section{NUMERICAL CALCULATIONS}

Numerical simulations were carried out to compare the results of the three model systems whose responses have been formulated in the preceding sections. The aim of the simulations was three-fold: First to investigate the response of a region of scatterers with a single realization of scatterer locations such as might be measured in an experimental system, second to validate the ensemble averaged limit for such scattering responses and finally, to validate the effective medium model as a representation of that ensemble-average scattering response. Our ultimate aim is to identify whether, and under what conditions, a simple effective medium model could be used to reproduce the effects of backscatter for cavities in a solid medium.

Calculations were executed in MATLAB ${ }^{47}$ using double precision complex arithmetic. By their nature, the calculations were done in discrete-time, discrete-frequency space. The sampling frequency applied in the simulations was $50 \mathrm{MHz}$ over a record length of 1024 samples, giving a time-domain resolution of $20 \mathrm{~ns}$ and a window length of $20.48 \mu \mathrm{s}$. All calculations of the system response under models A-C were carried out in the frequency domain, and were converted into simulated transducer signals by convolution with a typical transducer response. The signal used as input to the simulations was that obtained experimentally using a pair of identical transducers of $10 \mathrm{MHz}$ center frequency (c.f.) (V311-SU, Olympus NDT, Waltham, USA) in a pitchcatch arrangement with $25 \mathrm{~mm}$ path length through water. The transducer waveform was digitized initially at $400 \mathrm{MHz}$ using a LeCroy 9450A oscilloscope (LeCroy Corp., Chestnut Ridge, NY) and then subsampled down to the simulation sampling frequency of $50 \mathrm{MHz}$. An additional simulated transducer transmit-receive response with a center frequency of around $5 \mathrm{MHz}$ was obtained by subsampling the measured (10 $\mathrm{MHz}$ c.f.) response in the frequency domain. Figure 3 shows the transmit-receive responses in the time domain for the initial measurement with the $10 \mathrm{MHz}$ transducer and the subsampled $5 \mathrm{MHz}$ result. Results in the time domain were obtained by Fourier transformation. For the purpose of

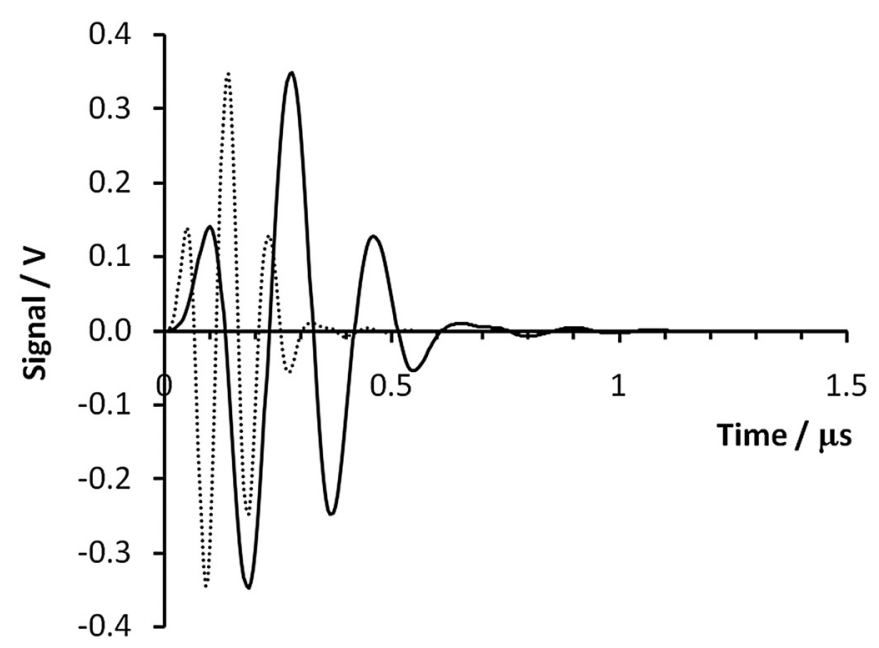

FIG. 3. Transmit-receive time-domain response for the simulated transducer signals with center frequencies $10 \mathrm{MHz}$ (dotted) and $5 \mathrm{MHz}$ (solid). 
TABLE I. Physical properties of the composite matrix materials used in calculations.

\begin{tabular}{ll}
\hline \hline Sound speed (longitudinal) & $3035 \mathrm{~m} \mathrm{~s}^{-1}$ \\
Density & $1564 \mathrm{~kg} \mathrm{~m}^{-3}$ \\
Shear modulus & $3.6 \mathrm{GPa}$ \\
Attenuation & 0 \\
\hline
\end{tabular}

graphical clarity, all of the time-domain functions shown on the results which follow have been up-sampled to $200 \mathrm{MHz}$ using a conventional $\sin x / x$ interpolation; they have been time-shifted so that the temporal origin coincides with the first received signal $\left(2 z_{\min } / c\right)$.

The simulations required intensive use of numerical integration of functions which contained oscillatory components with, potentially, nonzero and varying baselines. These were done using the MATLAB function "quadgk," which is optimized for oscillatory integrands and which has proved to be more accurate than the standard MATLAB functions "quad" and "quadl." In addition, the accuracy of integration is strongly affected by the way the integrand is expressed, and so all integrands were cast in forms of unity order to achieve maximum accuracy. The MATLAB pseudorandom number generator function "rand," based on the Mersenne Twister algorithm, ${ }^{48}$ was used to generate scatterer positions when considering randomly placed individual scatterers. The pairs of coordinates $(R, z)$ were generated from two sets of uniformly distributed values taken from the same pseudo-random number stream (same seed). The $z$ values were distributed uniformly over the layer thickness, whereas the $R$ coordinate values were calculated to obtain uniform scatterer distribution over the area, thus $R=R_{\max } \sqrt{x}$, where $x$ is the uniformly distributed random number. When using different numbers of scatterers, the smaller ensembles are subsets of the larger ensembles-a new random position generation was not used for each ensemble.

The medium modeled is a representation of an epoxybased composite with carbon reinforcing fibers, typical of aerospace applications. The composite is considered to be a homogeneous material for the purposes of these calculations, and its properties have been estimated from its constituents, shown in Table I. The shear modulus was estimated by specifying the ratio of longitudinal:shear wave speed to be $2: 1$. For the purposes of the present calculations, the attenuation of longitudinal waves in the composite was taken to be zero, in order to explore the physical aspects of the backscatter

TABLE II. The system parameters used in the calculations.

\begin{tabular}{ll}
\hline \hline Distance $z_{\min }$ & $2 \mathrm{~mm}$ \\
Layer thickness & $1 \mathrm{~mm}$ \\
Radius of scatterer region $R_{\max }$ & $20 \mathrm{~mm}$ \\
Cavity radius (spheres) & $10 \mu \mathrm{m}$ \\
Volume fraction of cavities & $1 \%$ \\
Transducer center frequencies & $10 \mathrm{MHz}, 5 \mathrm{MHz}$ \\
Sampling frequency & $50 \mathrm{MHz}$ \\
Number of samples & 1024 \\
\hline \hline
\end{tabular}

and effective medium models independently of the expected viscoelastic losses characteristic of resin polymer matrices.

The parameters of the system configuration are summarized in Table II, and were chosen to represent a typical ultrasonic measurement on composite with a 5 or $10 \mathrm{MHz}$ centerfrequency transducer, measuring at a depth of $2 \mathrm{~mm}$; this is the distance from the transducer surface to the front of the scatterer region. The radius of the spherical cavities is $10 \mu \mathrm{m}$. The thickness of the region in which scatterers are distributed, or of the layer of the effective medium, is $1 \mathrm{~mm}$. Since we are considering the scattering problem in the long wavelength limit, the order $n$ of the partial waves can be limited and we have set $n_{\max }=2$. Here the wavelength at $10 \mathrm{MHz}$ is $\sim 300 \mu \mathrm{m}$ and the cavity radius $10 \mu \mathrm{m}$.

\section{RESULTS}

\section{A. Discrete scatterer model A}

First we explore the system response for a small number of cavities present in the defined region, using model $\mathrm{A}$, the discrete scatterer model. The frequency response for a single cavity is shown in Fig. 4, and varies as the square of frequency, through the far-field amplitude for the cavity, $f(\theta)$, Eqs. (8), (2)-(4). When two cavities are present in the system (at randomly selected locations), the signals from the two cavities interfere with each other, resulting in interference peaks in the frequency response, overlaid on the trend in the square of frequency due to the scattering amplitude. Increasing the number of cavities to 1000 (at random locations within the defined region) results in a number of spikes and nodes in the frequency response on Fig. 4, which are not regularly spaced. These features are the consequence of the interference between signals from each pair of scatterers (cavities). However, the undulating pattern is still superimposed on the underlying trend in the square of frequency due to the scattering amplitude. The amplitude of the peaks also increases with frequency for the same reason, from the constructive interference between pairs of scatterers. The

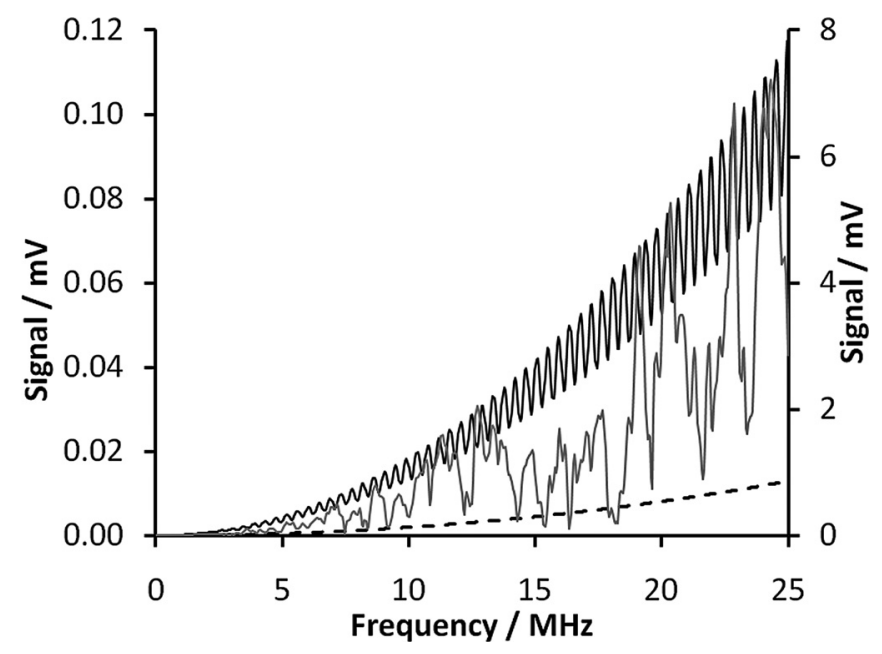

FIG. 4. Frequency-domain response for a small number of discrete spherical cavities of $10 \mu \mathrm{m}$ radius in the defined region; single cavity (dashed, black), two cavities (solid, black), 1000 cavities (solid, gray, right hand axis). 


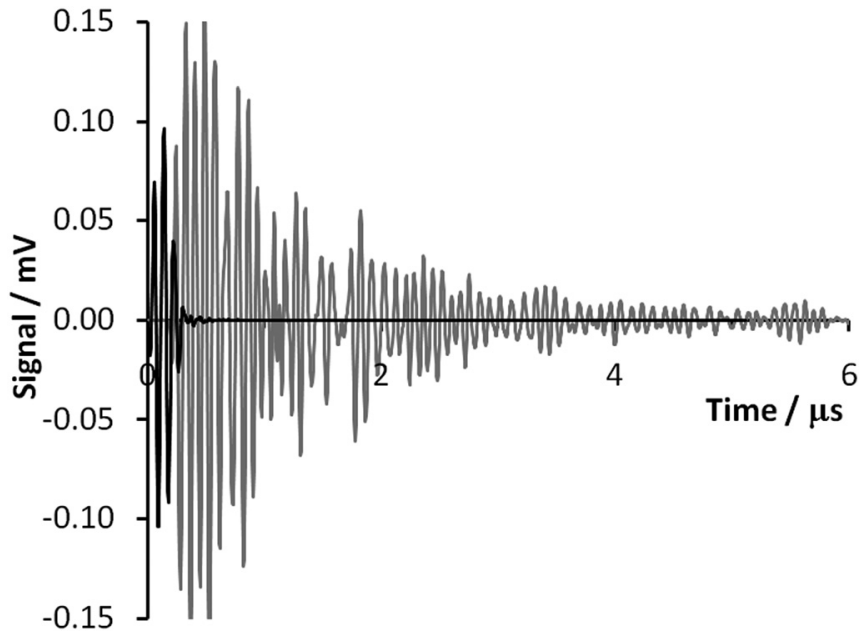

FIG. 5. Time-domain response at $10 \mathrm{MHz}$ for a single cavity (black) and 1000 cavities (gray).

corresponding time-domain response with a $10 \mathrm{MHz}$ center frequency transducer, shown in Fig. 5, is a long duration signal, with apparently random amplitude, relating to the signals received from the multiple cavities. In contrast, the response from a single cavity is almost identical to the transmitted signal.

Having identified how the interference between scattered fields causes irregular fluctuations in the frequency response, with an underlying trend in the square of frequency due to the scattering amplitude, we now show the response for a larger number of discrete scatterers, at concentrations by volume ranging from $1 \%-20 \%$. The corresponding number of cavities located in the defined region is from $3 \times 10^{6}$ $(1 \% \mathrm{v} / \mathrm{v})$ to $60 \times 10^{6}(20 \% \mathrm{v} / \mathrm{v})$ cavities. We reiterate here that each simulation with the discrete scatterer model $\mathrm{A}$ is with a single realization of scatterer positions. As the concentration is increased, more scatterers were added to the system, retaining those already present; thus the response at $1 \%$ is obtained for a subset of the cavities modeled at $20 \%$ concentration. Figure 6(a) shows the frequency response at concentrations of $1 \%$ and $2 \%$ by volume. The same features as already seen with 1000 scatterers can be identified in the response: A trend in the square of frequency, with overlaid random waviness, whose amplitude also increases with frequency. However, another trend is beginning to emerge; at the lowest frequencies (up to around $5 \mathrm{MHz}$ ) clear resonance peaks can be seen, with the peak amplitude independent of frequency. At higher concentrations $(10 \%$ and $20 \%$ by volume), Fig. 6(b), the increasing amplitude of the response at higher frequency seems to be less than the previous trend in the square of frequency, and the undulations are of lower amplitude. In addition, the emerging trend at the lower frequencies for resonance peaks with an amplitude independent of frequency, extends to higher frequency, almost $10 \mathrm{MHz}$ in this case. Note that the responses have been scaled in amplitude by the scatterer concentration for ease of comparison.

Similar resonance peaks occur in the response from a layer of homogeneous material due to interference between reflections from its front and back interfaces. What is seen from the results of the discrete scatterer model is the effect (a)

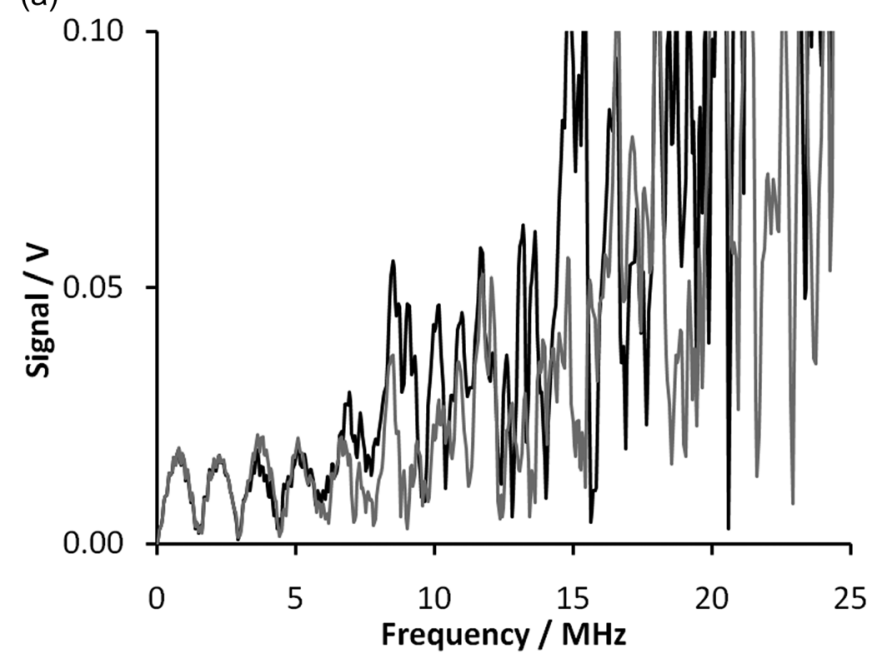

(b)

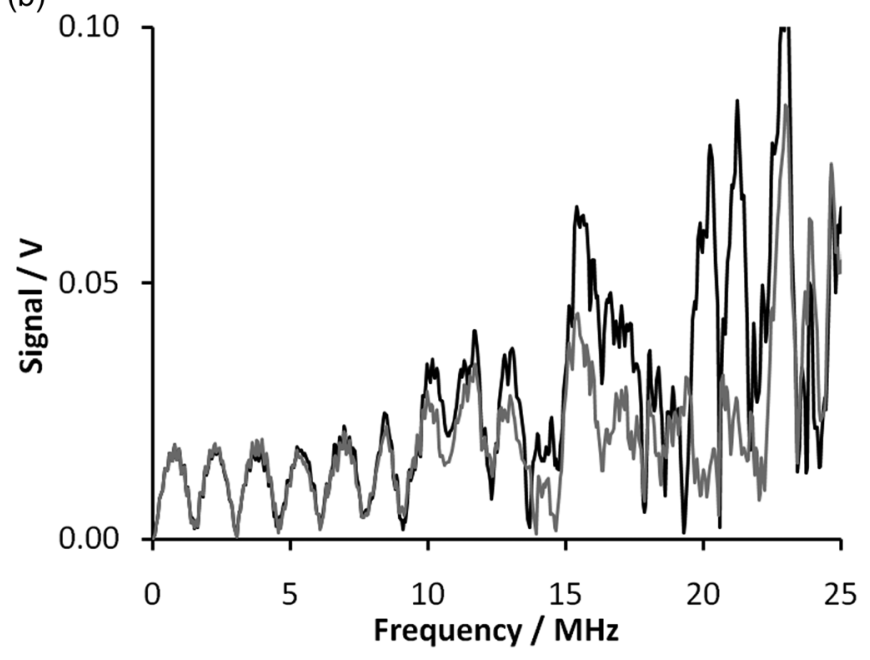

FIG. 6. Frequency response from the discrete scatterer model with varying concentrations of cavities (a) at $1 \%$ (black) and $2 \%$ (gray) concentration by volume, (b) at $10 \%$ (black) and $20 \%$ (gray) concentration by volume, scaled by volume fraction to facilitate comparison.

of the interference between scattered signals from individual cavities, leading to a response which has similar features to that of a homogeneous layer. Interestingly, the interference effects transform a trend in the square of frequency, to resonance peaks with amplitude independent of frequency.

In the time domain, the received signals with a $10 \mathrm{MHz}$ center frequency transducer are shown in Fig. 7(a), and for the $5 \mathrm{MHz}$ case in Fig. 7(b). At $10 \mathrm{MHz}$ [Fig. 7(a)], with $1 \%$ $\mathrm{v} / \mathrm{v}$ concentration of cavities, the time-domain signal is apparently random apart from the vestigial oscillatory response of the transducer; it is formed from the addition of signals from many cavities. At the higher concentration of $20 \%$, the signal has two main wave packets, suggesting an emerging layer-like behavior, but the second of the packets is of larger amplitude than the first, resulting from incomplete destructive interference between scattered signal components within the layer (the incoherent signal). The resonance peaks in the frequency domain response [Fig. 6(b)], even at 20\% concentration, did not extend as far as $10 \mathrm{MHz}$, so the layer-like behavior is not complete at this frequency. In contrast, in the 

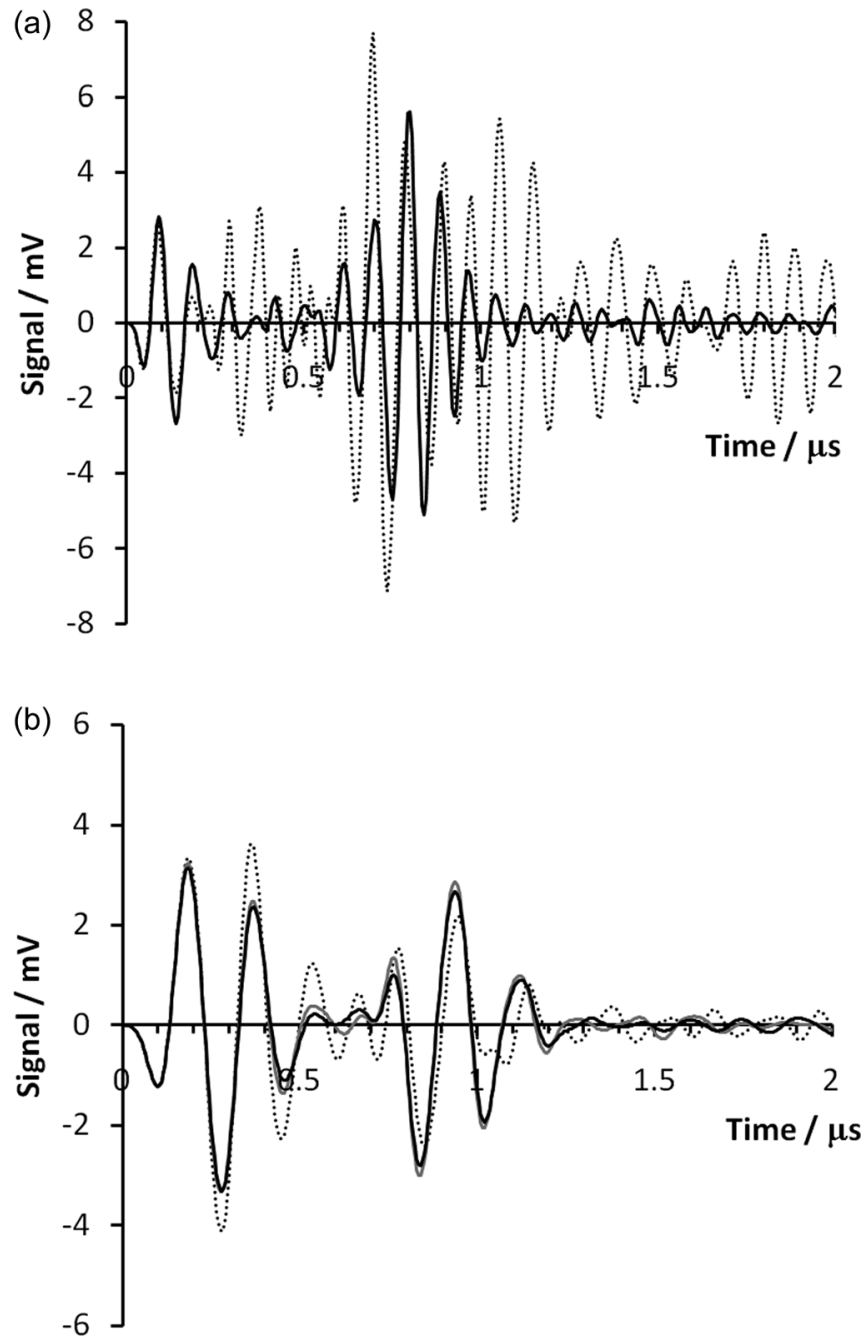

FIG. 7. Time-domain response from the discrete scatterer model with varying concentrations of cavities (a) with $10 \mathrm{MHz}$ center frequency transducer at $1 \% \mathrm{v} / \mathrm{v}$ (dotted, black) and $20 \% \mathrm{v} / \mathrm{v}$ (solid, black) concentration (b) with $5 \mathrm{MHz}$ center frequency transducer at 1\% (dotted, black), 10\% (solid, gray), and $20 \%$ (solid, black) concentration.

$5 \mathrm{MHz}$ case, the time-domain responses clearly have features of a homogeneous layer, Fig. 7(b). These features correspond to the resonance peaks in the frequency response seen in Fig. 6(a)-6(b); these are present at $5 \mathrm{MHz}$ at all concentrations shown. Even at a concentration of $1 \%$, the time-domain response has two clear wavepackets, similar to a front and back wall reflection. At $20 \%$ the waveform has a shape almost identical to the transmitted transducer signal, with inversion in the first received group, and not the second.

These numerical experiments using the discrete scatterer model have demonstrated a number of significant features. First, with only a small number of cavities, the response has a trend in the square of frequency, with interference effects causing peaks in the frequency domain, and a signal of randomly varying amplitude and long duration in the time domain. Second, at higher concentrations of cavities, the interference causes the emergence at the lowest frequencies of resonance peaks with amplitude independent of frequency. Third, as the concentration of cavities is increased, these resonance peaks appear up to higher frequencies.
Fourth, the time-domain response has similarities to that of a homogeneous layer if the transducer center frequency is below the upper limit of the region containing resonance peaks in the frequency response.

\section{B. Ensemble average model B}

The ensemble average model $B$ represents the response averaged over all possible realizations of scatterer positions; in our single-scattering approximation, this is taken to be equivalent to integration over uniformly distributed scatterers (see Sec. II B). One reason for the numerical calculation of the ensemble average result is to establish that it is indeed the limit of the summed signals from discrete scatterers as the number of scatterers is increased. The second is to establish its correspondence (as the numerically integrated limit of signals received from randomly distributed scatterers) with the effective medium model, which uses equivalent homogeneous properties. Our results for the ensemble average model $\mathrm{B}$ are shown in Fig. 8 in the frequency domain and Fig. 9 in the time-domain with the two different transducer signals.

Small amplitude oscillations on the frequency response of the ensemble average model at $1 \%$ due to the finite radius of the region containing scatterers (a diffraction effect, similar to edge waves) have been smoothed out by a moving average (Fig. 8); the corresponding delayed signal in the timedomain is beyond the range of the time-domain response shown (Fig. 9). We established that the radius of the region, $R_{\max }=20 \mathrm{~mm}$, was sufficient by calculating a second set of results from the ensemble average model B with a larger radius of $R_{\max }=25 \mathrm{~mm}$. We found no significant change in the smoothed response. Although the small oscillations were reduced in amplitude, they were still present, and appear to be slow to die away as $R_{\max }$ is increased. Hence the maximum radius of the region of $R_{\max }=20 \mathrm{~mm}$ was adequate for

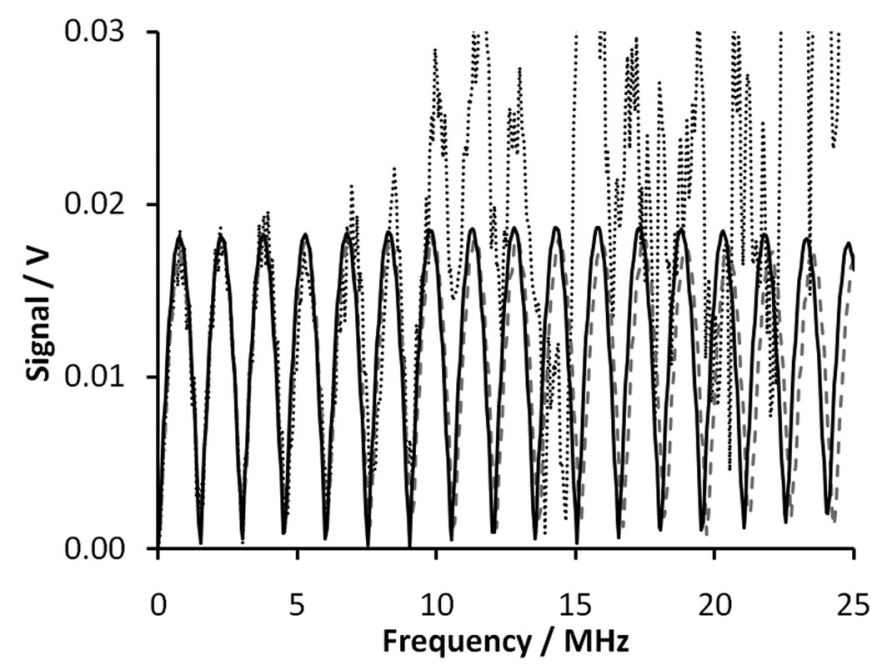

FIG. 8. Frequency response for ensemble average model B, smoothed by a three-point moving average (dashed, gray) and the effective medium model $\mathrm{C}$ (solid, black) at $1 \%$ volume fraction of cavities, and for the discrete scatterer model at $20 \%$ volume fraction of cavities (dotted, black), scaled by concentration for ease of comparison. 

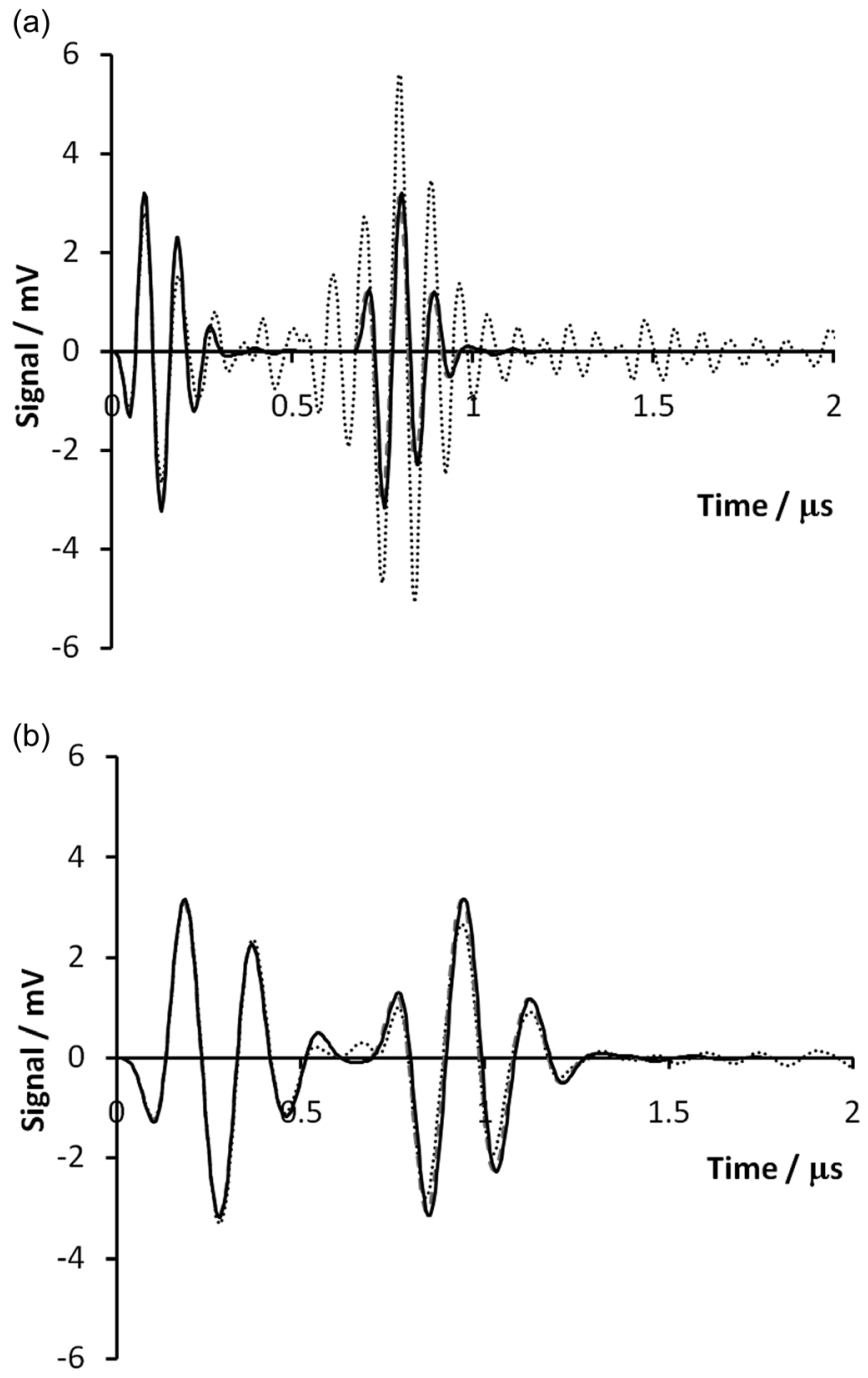

FIG. 9. Time-domain response for ensemble average model B (dashed, gray) and the effective medium model $\mathrm{C}$ (solid, black) at $1 \%$ volume fraction of cavities, and for the discrete scatterer model at $20 \%$ volume fraction of cavities (dotted, black), scaled by concentration for comparison) with (a) $10 \mathrm{MHz}$ center frequency transducer, (b) $5 \mathrm{MHz}$ center frequency transducer.

both the ensemble average and discrete scatterer models, B and $\mathrm{A}$, respectively.

In the frequency domain, the ensemble average response has regularly spaced resonance peaks over the entire frequency range, at intervals corresponding to the thickness of the defined region $(1 \mathrm{~mm})$. The amplitude of the peaks is almost independent of frequency. These features match those seen emerging at the lowest frequencies in the discrete scatterer model (Fig. 6), but here they are present over the entire frequency range. Hence, the squared frequency-dependence of the scattering amplitude for a single cavity is transformed into a frequency-independent resonance peak amplitude as a result of interference, in the ensemble average case. We saw for isotropic scatterers, for which an analytical result is obtainable, Eq. (26), that the ensemble average model predicts a frequency-independent reflection coefficient, except for the layer resonance effects, through the cancellation of the frequency-dependence of the scattering amplitude $f_{\text {iso }}$ by the $k^{2}$ term in the denominator.
A comparison of the results of the ensemble average model B with those of the discrete scatterer model $\mathrm{A}$ in the frequency domain (Fig. 8) demonstrates that at low frequencies, model $\mathrm{B}$ is indeed the correct limit for the scattered field for many discrete scatterers. However, at higher frequencies, the contributions of the scattered fields from individual scatterers result in sharply varying frequency response, as the interference effects are incomplete. It may be observed that the discrete scatterer model frequency response converges to the ensemble-average limit faster (i.e., at lower concentration) for lower frequencies. This effect will be further explored in Sec. VI.

In the time domain, Fig. 9, the system response has two signals separated in time, by an interval corresponding to the round-trip time in the layer. Thus, the average over scatterer positions results in a response in which all scattered fields destructively interfere except for those near the front and back boundaries; the response is similar to that of a homogeneous medium. The first signal is inverted relative to the transmitted signal, whereas the second is not, implying that the equivalent impedance of the layer is lower than the surrounding matrix. With a $10 \mathrm{MHz}$ center frequency transducer, Fig. 9(a), the discrete scatterer model at $20 \%$ concentration of cavities, agrees well with the ensembleaveraged result for the apparent front-face reflection, but is rather different for the second, back-face reflection. However, using a $5 \mathrm{MHz}$ center frequency transducer signal results in very good agreement between the discrete scatterer results and the ensemble-average model. We conclude that, although the ensemble-average model does indeed predict the response at sufficiently high concentration of scatterers (within the low concentration assumption required for the single scattering formulation), and sufficiently low transducer frequency, the actual response observed for a single realization of cavity locations may be far from the ensembleaverage predictions. We explore the conditions under which the ensemble average may be applied to a single realization of scatterers in Sec. VI.

\section{Effective medium model C}

The frequency response of the effective medium model, $\mathrm{C}$, which treats the region containing scatterers as an equivalent homogeneous medium, is also shown in Fig. 8. In close similarity to the ensemble-averaged model, $\mathrm{B}$, the resonance peaks due to the interference of signals reflected from the front and back interfaces of the region have amplitude which is almost independent of frequency. This is a result of the frequency-independent effective impedance, Eq. (23) obtained for the region from the effective medium models used. There are small differences in the resonance peak amplitudes and the location of the pseudonodes at higher frequencies. We attribute these to our assumption of an identical exciting field at each scatterer, equal to the incident wave from the transducer, which greatly simplified the numerical modeling. In Sec. III, we showed by analytical comparison of the models for isotropic scatterers, that the interference term in the response related to the effective wavenumber of the cavity-containing composite, $K$ in the effective medium 
model, but related to the wavenumber of the matrix, $k$ for the ensemble average model, resulting in a marginally different layer resonance frequency.

In the time domain, the effective medium model $\mathrm{C}$ and ensemble average model $\mathrm{B}$ are almost indistinguishable (Fig. 9). In comparison with homogeneous materials, we can now identify the inversion of the front face reflection as being associated with the lower impedance in the layer than in the matrix. The back-face reflection is not inverted, since it is reflected from an interface at which the impedance increases.

Our results have demonstrated numerically that the averaged response from randomly located scatterers (model B) is correctly modeled by an equivalent homogeneous medium with effective properties defined by the effective wavenumber and density in the medium. Since we have also shown that the ensemble average model B is the limit of the discrete scatterer response for a single realization of cavity positions, model A, under certain conditions, we can conclude that the effective medium model is an appropriate way to simulate the response of regions of scatterers, under the same conditions. We now explore those conditions under which the effective medium model is likely to be an accurate representation of the response from a cavity-filled matrix with only a single realization of cavity locations, such as would occur in a one-shot experimental measurement on a piece of composite material.

\section{DISCUSSION}

We have seen that the emergence of an effective medium-style response occurs, in general terms, at low frequencies, and high concentrations of cavities. In order to quantify these conditions, we must consider the length-scale at which the medium containing cavities can be considered to be homogeneous. The inhomogeneity of the medium is on a length scale comparable with the average intercavity distance. When the wavelength is much larger than this length scale (i.e., at low frequencies), the material appears as effectively homogeneous, and the response approaches the ensemble-averaged or effective-medium result. At high frequencies, the wavelength is shorter than or comparable with the intercavity distance, and the material shows characteristics of an inhomogeneous medium, in which the scattered fields from each individual cavity can be identified in the signal.

Thus, we can express the condition for the effective medium/ensemble average limit to be valid as

$$
f<f_{\max }=c /(b l),
$$

where $l=\left(4 \pi a^{3} / 3 \phi\right)^{1 / 3}$ is the average intercavity spacing, and $f$ is the frequency, $c$ is the wave speed, $a$ is the cavity radius, and $\phi$ is the volume fraction of cavities. The constant $b$ is effectively the average number of scatterers per wavelength above which an ensemble average model is a valid representation of a single realization of scatterer positions. We have estimated the value of $b$ by comparing the frequency domain results of models $\mathrm{A}$ and $\mathrm{B}$ and noting the frequency at which they differed by $10 \%$. A plot of these frequencies versus scatterer concentration was then compared with expectation on the basis of Eq. (27). This procedure gave a value of $b=12$; that is to, say, the ensemble average model becomes valid at frequencies and scatterer concentrations which yield an average scatterer density of 12 scatterers per wavelength.

In general terms, $f_{\max }$ is higher at higher concentrations [see Fig. 6(a)-6(b), 7(a)-7(b)]; alternatively, at any given frequency, the concentration must be sufficiently large to satisfy Eq. (27) (see Fig. 6).

The effective medium criterion, Eq. (27), also predicts that a smaller radius (which leads to an increase in the number of scatterers for a given volume fraction) extends the frequency range for the validity of the effective medium model. Although the effect of cavity radius was not explored in this numerical study, we anticipate that using a smaller radius would have produced agreement with the effective medium model over a wider range of frequency. However, it would also have required many more cavities to be modeled in the discrete scatterer model $\mathrm{A}$, and the radius was chosen so as to restrict the number of cavities in the region to achieve a manageable simulation.

This simple expression, Eq. (27), provides a guideline for the conditions under which the effective medium model should be a good representation of the response of the cavity-containing medium, for a single realization of cavity locations. It is physically based, and agrees with the trends observed in our numerical simulations.

\section{CONCLUSIONS}

We have used an interpretation of the Rayleigh method to investigate the ultrasonic pulse-echo responses that might occur in the NDE of composites which contain porosity flaws. A stochastic discrete scatterer model was shown to tend to agreement with ensemble average and equivalent homogeneous medium models at higher concentrations of porous inclusions, thus providing a numerical validation of the equivalent homogeneous properties. A criterion has been established which sets the maximum frequency at which either ensemble average or equivalent medium models can represent the pulse-echo behavior of a field of porous inclusions. This frequency increases as the interpore spacing reduces, or equivalently, as pore size reduces and/or pore volume fraction increases. A similar approach would be valid for other scatterer shapes, such as microscopic cylindrical pores and ribbons.

The significant implication of this work is that the ensemble average or equivalent homogeneous medium approximations could be used in models of ultrasonic wave propagation in composites which contain porosity flaws, under certain conditions of frequency, pore size, and pore concentration. This overcomes the difficulties associated with the inclusion of scattering phenomena in stratified medium propagation models, and will therefore enable simulations of ultrasonic NDE procedures which are physically realistic, and which can be used to gain understanding of the process of porosity flaw detection and characterization. 
${ }^{1}$ B. Hosten and M. Castaings, "Transfer-matrix of multilayered absorbing and anisotropic media-Measurements and simulations of ultrasonic wave-propagation through composite-materials," J. Acoust. Soc. Am. 94(3), 1488-1495 (1993).

${ }^{2}$ A. H. Nayfeh and D. E. Chimenti, "Propagation of guided-waves in fluidcoupled plates of fiber-reinforced composite," J. Acoust. Soc. Am. 83(5), 1736-1747 (1988).

${ }^{3}$ L. Wang and S. I. Rokhlin, "Ultrasonic wave interaction with multidirectional composites: Modeling and experiment," J. Acoust. Soc. Am. 114(5), 2582-2595 (2003).

${ }^{4}$ W. T. Thomson, "Transmission of elastic waves through a stratified medium,” J. Appl. Phys. 21, 89-93 (1950).

${ }^{5}$ L. Knopoff, "A matrix method for elastic wave problems," B. Seismol. Soc. Am. 43, 17-34 (1964).

${ }^{6} \mathrm{Z}$. Hashin, "Analysis of composite materials-A survey," J. Appl. Mech. 50, 481-505 (1983).

${ }^{7}$ B. G. Martin, "Ultrasonic attenuation due to voids in fibre-reinforced solids containing voids," J. Appl. Phys. 48(8), 3368-3373 (1977).

${ }^{8}$ L. B. Greszczuk, "Interfilamentary stresses in filamentary composites," AIAA J. 9(7), 1274-1280 (1971).

${ }^{9}$ S. M. Nair, D. K. Hsu, and J. H. Rose, "Porosity estimation using the frequency dependency of the ultrasonic attenuation,” J. Nondestruct. Eval. 8, 13-26 (1989).

${ }^{10}$ L. Adler, J. H. Rose, and C. Mobley, "Ultrasonic method to determine gas porosity in aluminum-alloy castings - Theory and experiment," J. Appl. Phys. 59(2), 336-347 (1986).

${ }^{11}$ M. J. Mienczakowski, A. K. Holmes, and R. E. Challis, "Modeling of ultrasonic wave propagation in composite airframe components," 34th Annual Review of Progress in Quantitative Nondestructive Evaluation (July 22-27, 2007), Golden, CO, in Review of Progress in Quantitative Nondestructive Evaluation, Vol $27 a$ and $27 b$, edited by D. O. Thompson and D. E. Chimenti (AIP, New York, 2008), Vol. 975, pp. 995-1001.

${ }^{12}$ J. D. Eshelby, "The determination of the elastic field of an ellipsoidal inclusion, and related problems," Proc. R. Soc. London, Ser. A 241, 376396 (1957).

${ }^{13}$ R. Hill, "A self-consistent mechanics of composite materials," J. Mech. Phys. Solids 13, 213-222 (1965).

${ }^{14} \mathrm{~B}$. Budiansky, "On the elastic moduli of some heterogeneous materials," J. Mech. Phys. Solids 13, 223-227 (1965).

${ }^{15} \mathrm{Z}$. Hashin and S. Shtrikman, "A variational approach to the theory of the elastic behaviour of multiphase materials," J. Mech. Phys. Solids 11, 127140 (1963).

${ }^{16}$ W. J. Parnell, I. D. Abrahams, and P. R. Brazier-Smith, "Effective properties of a composite half-space: exploring the relationship between homogenization and multiple scattering theories," Q. J. Mech. Appl. Math. 63(1), 1-32 (2010).

${ }^{17}$ F. J. Sabina and J. R. Willis, "A simple self-consistent analysis of wave propagation in particulate composites," Wave Motion 10, 127-142 (1988).

${ }^{18}$ S. K. Kanuan and V. M. Levin, "Effective medium method in the problem of axial elastic shear wave propagation through fiber composites," Int. J. Solids Struct. 40, 4859-4878 (2003).

${ }^{19}$ J.-Y. Kim, "Models for wave propagation in two-dimensional random composites: a comparative study," J. Acoust. Soc. Am. 127(4), 2201-2209 (2010).

${ }^{20}$ L. L. Foldy, "The multiple scattering of waves," Phys. Rev. 67(3-4), 107119 (1945).

${ }^{21}$ M. Lax, "Multiple scattering of waves," Rev. Mod. Phys. 23, 287-310 (1951).

${ }^{22}$ M. Lax, "Multiple scattering of waves II The effective field in dense systems," Phys. Rev. 85, 621-629 (1952).

${ }^{23} \mathrm{~V}$. Twersky, "On scattering of waves by random distributions I. Free space scatterer formalism," J. Math. Phys. 3, 700-715 (1962).

${ }^{24}$ P. C. Waterman and R. Truell, "Multiple scattering of waves," J. Math. Phys. 2, 512-537 (1961).

${ }^{25}$ J. G. Fikioris and P. C. Waterman, "Multiple scattering of waves II. 'Hole corrections' in the scalar case,” J. Math Phys. 5, 1413-1420 (1964).
${ }^{26}$ V. K. Varadan, Y. Ma, and V. V. Varadan, "A multiple scattering theory for elastic wave propagation in discrete random media," J. Acoust. Soc. Am. 77, 375-385 (1985).

${ }^{27}$ Y. C. Angel and C. Aristegui, "Analysis of sound propagation in a fluid through a screen of scatterers," J. Acoust. Soc. Am. 118(1), 72-82 (2005).

${ }^{28}$ J. M. Conoir and A. N. Norris, "Effective wavenumbers and reflection coefficients for an elastic medium containing random configurations of cylindrical scatterers," Wave Motion 47(3), 183-197 (2010).

${ }^{29}$ P. Y. Le Bas, F. Luppe, and J. M. Conoir, "Reflection and transmission by randomly spaced elastic cylinders in a fluid slab-like region," J. Acoust. Soc. Am. 117(3), 1088-1097 (2005).

${ }^{30} \mathrm{~A}$. Maurel, "Reflection and transmission by a slab with randomly distributed isotropic point scatterers," J. Comput. Appl. Math. 234, 1842-1850 (2010).

${ }^{31}$ C. Aristegui and Y. C. Angel, "Coherent sound propagation across the effective interfaces of an immersed screen," Wave Motion 47, 199-204 (2010).

${ }^{32}$ F. Luppe and J. M. Conoir, "Multiple scattering by cylinders randomly located in a fluid:effective properties," J. Phys.: Conf. Ser. 269, 012015 (2011).

${ }^{33}$ P. A. Martin, A. Maurel, and W. J. Parnell, "Estimating the dynamic effective mass density of random composites," J. Acoust. Soc. Am. 128(2), 571-577 (2010).

${ }^{34}$ C. M. Linton and P. A. Martin, "Multiple scattering by multiple spheres: A new proof of the Lloyd-Berry formula for the effective wavenumber," SIAM J. Appl. Math. 66(5), 1649-1668 (2006).

${ }^{35} \mathrm{P}$. Lloyd and M. V. Berry, "Wave propagation through an assembly of spheres IV Relations between different multiple scattering theories," Proc. Phys. Soc., London 91, 678-688 (1967).

${ }^{36}$ J. M. Conoir, S. Robert, A. El Mouhtadi, and F. Luppe, "Reflection and transmission at low concentration by a depth-varying random distribution of cylinders in a fluid slab-like region," Wave Motion 46(8), 522-538 (2009).

${ }^{37}$ J. Dubois, C. Aristegui, O. Poncelet, and A. L. Shuvalov, "Coherent acoustic response of a screen containing a random distribution of scatterers: comparison between different approaches," J. Phys.: Conf. Ser. 269, 012004 (2011).

${ }^{38}$ J.W.S. Strutt (Baron Rayleigh), The Theory of Sound, Reprint of 1894 2nd ed. (Dover, New York, 1945), Chap. XVII, pp. 272-284.

${ }^{39} \mathrm{P}$. S. Epstein and R. R. Carhart, "The absorption of sound in suspensions and emulsions. I. Water fog in air," J. Acoust. Soc. Am. 25(3), 553-565 (1953).

${ }^{40}$ J. R. Allegra and S. A. Hawley, "Attenuation of sound in suspensions and emulsions: theory and experiments," J. Acoust. Soc. Am. 51, 1545-1564 (1972).

${ }^{41}$ C. F. Ying and R. Truell, "Scattering of a plane longitudinal wave by a spherical obstacle in an isotropically elastic solid," J. Appl. Phys. 27, 1086-1097 (1956).

${ }^{42}$ R. E. Challis, J. S. Tebbutt, and A. K. Holmes, "Equivalence between three scattering formulations for ultrasonic wave propagation in particulate mixtures," J. Phys. D: Appl. Phys. 31(24), 3481-3497 (1998).

${ }^{43}$ R. E. Challis, M. J. W. Povey, M. L. Mather, and A. K. Holmes, "Ultrasound techniques for characterizing colloidal dispersions," Rep. Prog. Phys. 68(7), 1541-1637 (2005).

${ }^{44}$ Maple version 11.01, Waterloo Maple Inc., Waterloo, Canada.

${ }^{45}$ P. M. Morse and H. Feshbach, Methods of Theoretical Physics (McGrawHill, New York, 1953), Pt. II, p. 1065.

${ }^{46}$ R. E. Challis, R. J. Freemantle, J. D. H. White, and G. P. Wilkinson, "Ultrasonic compression wave NDT of adhered metal lap joints of uncertain dimensions," Insight 37(12), 954-963 (1995).

${ }^{47}$ MATLAB $^{\circledR}$ version R2008a (v. 7.6.0.324), The Mathworks Inc., Natick, MA (2008).

${ }^{48}$ H. Matsumoto and T. Nishimura, "Mersenne Twister: A 623-dimensionally equidistributed uniform pseudorandom number generator," ACM Trans. Model. Comput. Simul. 8(1), 3-30 (1998). 